\title{
COLEGIO INTERNACIONAL DE CIRUJANOS: POSICIONANDO AL PERÚ A NIVEL MUNDIAL
}

\author{
INTERNATIONAL COLLEGE OF SURGEONS: POSITIONING PERU AT WORLD LEVEL \\ Manuel Huamán-Guerrero 1,a,b,c, Jhony A. De La Cruz-Vargas 2,d,e,f
}

\section{INTERNATIONAL COLLEGE OF SURGEONS \\ $X$ CURSO INTERNACIONAL DE AVANCES EN MEDICINA CIENTÍFICA, TECNOLÓGICA Y QUIRÚRGICA}

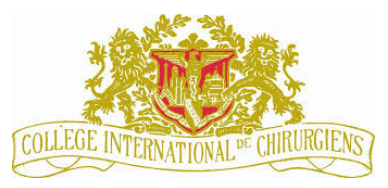

Con gran éxito se realizó en la explanada de la Universidad Ricardo Palma, el: X Curso Internacional de Avances en Medicina Científica, Tecnológica y Quirúrgica y la IX Conferencia de Educación Medica e Investigación. Dicho evento internacional tuvo lugar los días 10,11 y 12 de noviembre del presente año, y contó con la asistencia de más de 700 participantes de las diversas especialidades médicas, docentes universitarios y estudiantes de medicina.

Los invitados nacionales e internacionales presentaron sus experiencias y conferencias, sobre los últimos avances en las diversas áreas científicas, tecnológicas y quirúrgicas, así como en temas de educación médica e investigación. Destacamos la distinguida participación de nuestros conferencistas internacionales:

- Prof. Dr. Mark D. Duncan, Johns Hopkins Hospital, USA

- Prof. Dr. Yik Hong Ho, Cook University, Australia

- Prof. Dr. Miguel Pérez, Salud Publica, California, USA

- Prof. Dr. Flavio Takeda, Universidad de Sao Paulo, Brasil

- Prof. Dr. Guido Parket, Paraguay

- Prof. Dr. Raul Sebastian, Johns Hopkins Hospital, USA

- Prof. Max Downham, Chicago, USA

- $\quad$ Prof. Dra. Aij-Lie Kwan, Hong Kong, china

Un momento central fue la Ceremonia de Inauguración, que conto previamente con una conferencia del Sr. Rector de la Universidad Ricardo Palma, el Dr. Iván Rodríguez, y luego ante autoridades internacionales del Colegio y autoridades académicas nacionales se realizó la Juramentación del nuevo Presidente Mundial del Colegio Internacional de Cirujanos (ICS:https://www.icsglobal.org), el Prof. Dr. Manuel Huamán Guerrero, quien asumió sus funciones pocas semanas atrás en el Congreso de Kyoto, en Japón, y es a partir de diciembre de 2016, el flamante Presidente de este prestigioso Colegio Internacional. Es altamente destacable que un médico cirujano peruano, ocupe este lugar, y es un verdadero orgullo como país y como Institución, tener profesionales de esta magnitud y prestigio a nivel internacional. El Dr. Manuel Huamán se ha desempeñado como Decano de la Facultad de Medicina Humana desde sus inicios, y es actualmente el Vicerrector Académico de la Universidad Ricardo Palma.

En temas de investigación, se presentaron temas de suma actualidad en el área biomédica y quirúrgica, se contó con más de 40 posters de investigación científica, y se entregaron premios en 4 categorías: Mejor poster de Investigación Básica, Clínica, Innovación y Epidemiológica.

Para los organizadores de este evento: el Colegio Internacional de Cirujanos, la Facultad de Medicina Humana y la Universidad Ricardo Palma, fue muy satisfactorio ver el interés, la asistencia y la participación de los asistentes.

Además de los objetivos de actualización e intercambio de experiencias, el evento contribuyo a desarrollar y establecer vinculaciones con centros internacionales para avanzar en temas educativos y proyectos de investigación, estos beneficiaran tanto a estudiantes como a docentes universitarios.

\footnotetext{
'Vicerrector Académico de la Universidad Ricardo Palma.

a Director General del INICIB.

bEditor en Jefe de la Revista de la Facultad de Medicina Humana.

- Doctor en Medicina y Especialista en Cirugía Oncológica.

2 Director Adjunto del INICIB.

Coeditor de la Revista de la Facultad de Medicina Humana.

e Doctor en Medicina, Maestría en Investigación Clínica.

Especialista en Oncología Médica.
}

Citar como: Manuel Huamán-Guerrero, Jhony A. De La Cruz-Vargas. Colegio Internacional de Cirujanos: Posicionando al Perú a nivel mundial [Editorial]. Rev. Fac. Med. Hum. 2016;16(3):12. DOI 10.25176/RFMH.v16.n3.647 